\title{
TRANEXAMIC ACID AND TRANSFUSION REOUIREMENTS IN PRIMARY AND REVISION TOTAL KNEE ARTHROPLASTY
}

\section{Catarina Marques ${ }^{1}$, Renata Gouveia ${ }^{1}$, Filipa Carioca ${ }^{1}$, Rita Poeira ${ }^{1}$}

1. Centro Hospitalar Lisboa Central

\section{BACKGROUND AND GOAL OF STUDY}

Tranexamic acid (TXA) appears to reduce perioperative blood loss and transfusion requirements in major orthopaedic surgery. In our hospital, a protocol for TXA administration in elective primary total knee arthroplasty (PTKA) and revision total knee arthroplasty (RTKA) was implemented. It consists of TXA $1 \mathrm{~g}$ diluted in $50 \mathrm{~mL}$ saline or $2 \mathrm{~g}$ in $100 \mathrm{~mL}$ saline administered through the drain after closure of the articular capsule. If bleeding exceeds $500 \mathrm{~mL}, 1 \mathrm{~g}$ of intravenous TXA or a $1 \mathrm{mg} / \mathrm{kg} / \mathrm{h}$ perfusion for $6 \mathrm{~h}$ should be administered 3 to 12 hours after surgery. Anaesthesiologists decided on TXA administration in accordance with their clinical judgement and protocol's exclusion criteria.

Our goal was to investigate if haemoglobin $(\mathrm{Hb})$ variation and transfusion requirements decreased with protocol implementation.

\section{MATERIALS AND METHODS}

Retrospective cohort study of all patients submitted to PTKA and RTKA during an one-year period at a single institution.

Exclusion criteria were no postoperative haemogram, incomplete medical records and dosing, timing or route of TXA administration not according to protocol.

Demographic data was obtained from a review of individual medical records. Specific information identified through retrospective chart reviews was included: administration of TXA, pre and postoperative $\mathrm{Hb}$ levels and postoperative transfusion records.

We compared transfusion requirements and postoperative $\mathrm{Hb}$ drop in the TXA group vs the No TXA group in PTKA and RTKA patients.

Statistical analysis was performed with SPSS version 22. A p-value $<.05$ significance level was used for all hypothesis tests. Fisher's exact test, chi-square test and $U$ Mann-Whitney test were employed when appropriate.

\section{RESULTS}

- 323 total knee arthroplasties, with 277 patients meeting our inclusion criteria:

- PTKA in 254 patients (Image 1)

- RTKA in 23 patients (Image 2)
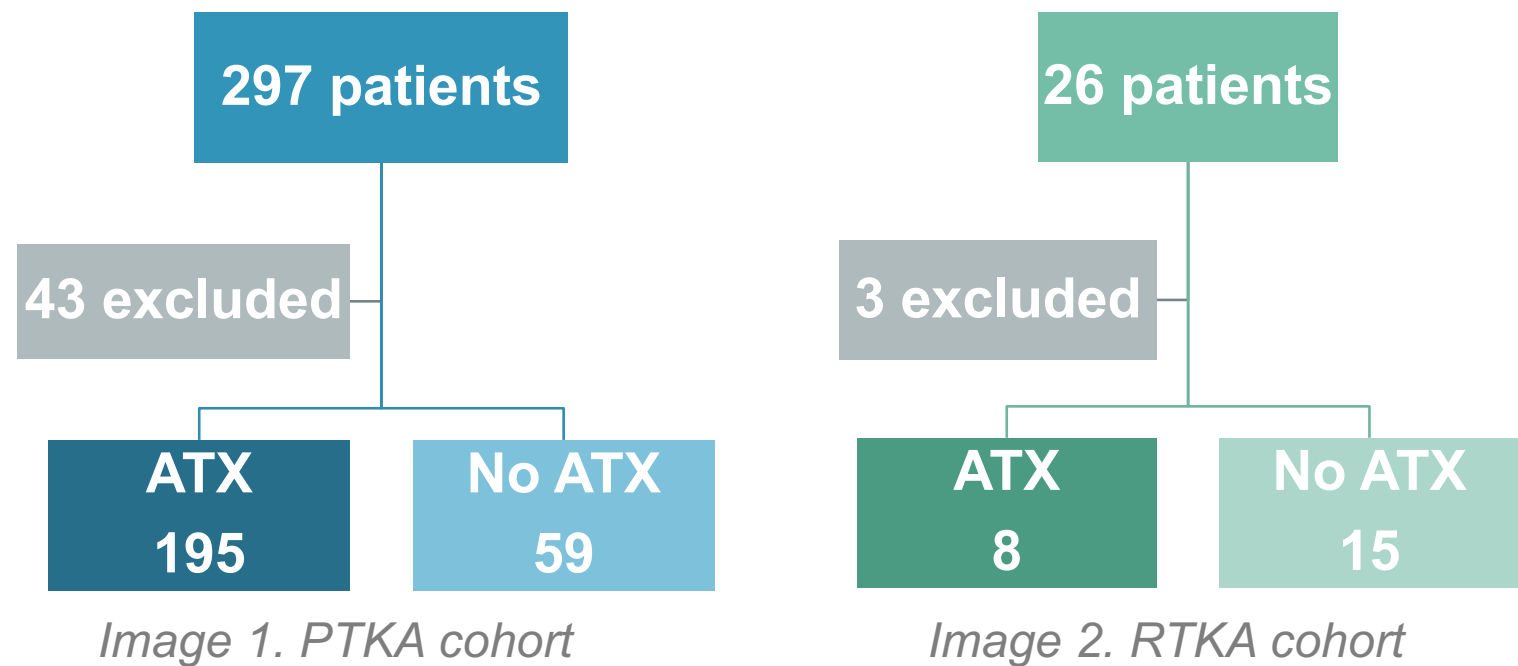

- 46 patients excluded (Table 1):

\begin{tabular}{|l|c|c|}
\hline Exclusion criteria & PTKA & RTKA \\
\hline Dosing & 10 & 0 \\
\hline Route of administration & 9 & 3 \\
\hline Unknown TXA dose & 18 & 0 \\
\hline No postoperative hemogram & 6 & 0 \\
\hline Table 1. Exclusion criteria & & \\
\hline
\end{tabular}

\section{PRIMARY TOTAL KNEE ARTHROPLASTY}

Demographic data:

- Sex $-72,8 \%$ female

- Mean age $-70 \pm 8$ years

- ASA physical status - 78,3\% ASA 2

- Anaesthetic technique $-73,6 \%$ spinal block

- Mean preoperative $\mathrm{Hb}-13,3 \pm 1,3 \mathrm{~g} / \mathrm{dL}$ (range $8,5-17,7$ )

Outcomes of TXA administration (Table 2):

\begin{tabular}{|c|l|c|c|c|c|}
\hline $\begin{array}{c}\text { Postoperative } \\
\text { Hb }\end{array}$ & Mean & $10,9 \pm 1,4$ & $9,4 \pm 1,4$ & $10,4 \pm 1,5$ & \\
& Range & $7,3-15,1$ & $5,5-12,6$ & $5,5-15,1$ & $<.001$ \\
\hline \multirow{2}{*}{ Hb variation } & Mean & $2,7 \pm 1,2$ & $3,8 \pm 1,5$ & $3 \pm 1,3$ & \\
& Range & $0,1-6,7$ & $1,3-8,6$ & $0,1-8,6$ & $<.001$ \\
\hline $\begin{array}{c}\text { RBC } \\
\text { transfusions }\end{array}$ & Yes & 11 & 18 & 29 & \\
\hline
\end{tabular}

Table 2. Outcomes of TXA administration in PTKA

- Mean postoperative $\mathrm{Hb}$ drop - significantly lower in TXA group vs. No TXA group $(2,7 \pm 1,2 \mathrm{~g} / \mathrm{dL}$ vs. $3,8 \pm 1,5 \mathrm{~g} / \mathrm{dL}, \mathrm{p}<.001)$.

- Transfusion rate - significantly lower in TXA group vs. No TXA group $(5,6 \%$ vs. $30,5 \% ; p<.001)$

- Total number of red blood cell units (RBCU) transfused significantly lower $(p<.001)$ in TXA group vs. No TXA group (16 vs. 33 RBCU transfused, max. 3 vs. 6 units per patient)

\section{REVISION TOTAL KNEE ARTHROPLASTY}

Demographic data:

- Sex $-60 \%$ female

- Mean age $-70 \pm 16$ years

- ASA physical status - 54\% ASA 3

- Anaesthetic technique $-72 \%$ general anaesthesia

- Mean preoperative $\mathrm{Hb}-12,4 \pm 8$ (range 7,5 - 17,6)

Outcomes of TXA administration (Table 3):

\begin{tabular}{|c|l|c|c|c|c|c|}
\hline \multirow{2}{*}{$\begin{array}{c}\text { Postoperative } \\
\text { Hb }\end{array}$} & Mean & $9,9 \pm 1,5$ & $9,2 \pm 1,8$ & $9,4 \pm 1,7$ & \multirow{2}{*}{ NS } \\
\cline { 2 - 6 } & Range & $7,0-11,3$ & $6,6-13,0$ & $6,-13$ & \\
\hline \multirow{2}{*}{ Hb variation } & Mean & $2,6 \pm 1,5$ & $2,9 \pm 1,4$ & $2,8 \pm 1,4$ & \multirow{2}{*}{ NS } \\
\cline { 2 - 6 } & Range & $0,7-5,9$ & $0,2-5,1$ & $0,2-5,9$ & \\
\hline RBC & Yes & 2 & 7 & 9 & \multirow{2}{*}{ NS } \\
\hline transfusions & No & 6 & 8 & 14 & \\
\hline
\end{tabular}

Table 3. Outcomes of TXA administration in RTKA

- Mean postoperative $\mathrm{Hb}$ drop and transfusion requirements were lower in TXA group vs. No TXA group, but the difference did not reach statistical significance $(2,6 \pm 1,5 \mathrm{~g} / \mathrm{dL}$ vs. $2,9 \pm 1,4$ $\mathrm{g} / \mathrm{dL}, \mathrm{p}=0,591 ; 33,3 \%$ vs. $46,7 \%, p=0,4)$.

\section{DISCUSSION \& CONCLUSIONS}

Administration of TXA in PTKA patients was associated with a significant reduction in postoperative haemoglobin drop, number of patients requiring $\mathrm{RBC}$ transfusions and number of $\mathrm{RBC}$ units transfused per patient. In RTKA patients, there was a trend towards reduction of blood transfusion requirements with the use of TXA, however sample size was too small to draw conclusions.

In conclusion, TXA IV administration in PTKA appears to reduce both postoperative haemoglobin variation and transfusion requirements, so its use in PTKA is recommend. A larger sample should be considered to investigate the role of TXA in RTKA. 